ARTICLE

DOI: $10.1038 / \mathrm{s} 41467-018-04017-\mathrm{x}$

\title{
Correlation of the Dzyaloshinskii-Moriya interaction with Heisenberg exchange and orbital asphericity
}

\author{
Sanghoon Kim¹,2, Kohei Ueda,3, Gyungchoon Go4, Peong-Hwa Jang ${ }^{4}$, Kyung-Jin Lee (i) 4,5, \\ Abderrezak Belabbes ${ }^{6}$, Aurelien Manchon (1) ${ }^{6}$, Motohiro Suzuki ${ }^{7}$, Yoshinori Kotani ${ }^{7}$, Tetsuya Nakamura ${ }^{7}$, \\ Kohji Nakamura ${ }^{8}$, Tomohiro Koyama9 ${ }^{9}$ Daichi Chiba (10 ${ }^{9}$, Kihiro. T. Yamada', Duck-Ho Kim', Takahiro Moriyama', \\ Kab-Jin Kim ${ }^{1,10}$ \& Teruo Ono ${ }^{1,11}$
}

Chiral spin textures of a ferromagnetic layer in contact to a heavy non-magnetic metal, such as Néel-type domain walls and skyrmions, have been studied intensively because of their potential for future nanomagnetic devices. The Dyzaloshinskii-Moriya interaction (DMI) is an essential phenomenon for the formation of such chiral spin textures. In spite of recent theoretical progress aiming at understanding the microscopic origin of the DMI, an experimental investigation unravelling the physics at stake is still required. Here we experimentally demonstrate the close correlation of the DMI with the anisotropy of the orbital magnetic moment and with the magnetic dipole moment of the ferromagnetic metal in addition to Heisenberg exchange. The density functional theory and the tight-binding model calculations reveal that inversion symmetry breaking with spin-orbit coupling gives rise to the orbitalrelated correlation. Our study provides the experimental connection between the orbital physics and the spin-orbit-related phenomena, such as DMI.

\footnotetext{
${ }^{1}$ Institute for Chemical Research, Kyoto University, Uji, Kyoto 611-0011, Japan. ${ }^{2}$ Department of Physics, University of Ulsan, Ulsan 44610, Korea.

${ }^{3}$ Department of Materials Science and Engineering, Massachusetts Institute of Technology, Cambridge, MA 02139, USA. ${ }^{4}$ Department of Materials Science \& Engineering, Korea University, Seoul 02841, Korea. ${ }^{5}$ KU-KIST Graduate School of Converging Science and Technology, Korea University, Seoul 02841, Korea. ${ }^{6}$ Physical Science and Engineering Division (PSE), King Abdullah University of Science and Technology (KAUST), Thuwal 23955-6900, Saudi Arabia. 7 Japan Synchrotron Radiation Research Institute (JASRI), Sayo, Hyogo 679-5198, Japan. ${ }^{8}$ Department of Physics Engineering, Mie University, Tsu, Mie 514-8507, Japan. ${ }^{9}$ Department of Applied Physics, The University of Tokyo, Bunkyo, Tokyo 113-8656, Japan. ${ }^{10}$ Department of Physics, Korea Advanced Institute of Science and Technology, Daejeon 34141, Korea. ${ }^{11}$ Center for Spintronics Research Network (CSRN), Graduate School of Engineering Science, Osaka University, Osaka 560-8531, Japan. These authors contributed equally: Sanghoon Kim, Kohei Ueda. Correspondence and requests for materials should be addressed to S.K. (email: sanghoon.kim@ulsan.ac.kr) or to T.O. (email: ono@scl.kyoto-u.ac.jp)
} 
C hiral interaction between two atomic spins owing to a strong spin-orbit coupling (SOC), which is known as the Dzyaloshinskii-Moriya interaction (DMI), has attracted intense interest ${ }^{1,2}$. In particular, it has been demonstrated that the DMI at the interface between ferromagnetic (FM) and nonmagnetic heavy metals (HMs) plays a major role for the formation of chiral spin textures, such as skyrmions ${ }^{3,4}$ and homochiral Néel-type domain walls $(\mathrm{DWs})^{5-7}$, which are attractive for the development of future information storage technology ${ }^{8}$. Understanding the microscopic origin of the DMI is indispensable for the realization of such chiral spin textures ${ }^{9,10}$. It has been reported that the proximity-induced magnetic moment in HM layers is critical to promote the $\mathrm{DMI}^{11}$. However, this proximity effect is still controversial because it has been also reported that the induced magnetic moment has no direct correlation with the $\mathrm{DMI}$ in the case of the Co/Pt system ${ }^{12,13}$. The scattering of spinpolarized electrons on spin-orbit coupled impurities is known to give rise to the DMI in spin-glass systems as a microscopic viewpoint ${ }^{14,15}$. However, the orbital hybridization between the spin-orbit coupled ions and the magnetic matrix was explicitly neglected in the theory, which becomes problematic when considering transition metal interfaces. On the other hand, theories have predicted that SOC combined with inversion symmetry breaking (ISB) naturally introduces a chirality to conduction electron spins in equilibrium and the interfacial DMI at an FM/ HM interface is related to this spin chirality ${ }^{16-20}$. It has also been reported that the spin chirality is a manifestation of the chirality of the orbital magnetism in strongly spin-orbit coupled systems with ISB ${ }^{21,22}$. These previous studies suggest a possible microscopic origin of the interfacial DMI, which has remained experimentally unaddressed so far.

Here we discuss the microscopic origin of the interfacial DMI with experimental and theoretical studies as follows: First, we show the temperature dependence of the DMI for a $\mathrm{Pt} / \mathrm{Co} / \mathrm{MgO}$ trilayer, which is one of the standard structures used for the studies of the $\mathrm{DMI}^{7,12,23}$, using the extended droplet model ${ }^{24}$. We find that the DMI increases with decreasing temperature in a range from 300 to $100 \mathrm{~K}$. In general, the electron-phonon interaction promotes thermally induced hopping between nearest neighbours when increasing the temperature ${ }^{25}$. As a result, it is expected that the difference between in-plane and out-of-plane hopping energies is reduced upon temperature increase.
Therefore, changing the temperature of the system allows for charge redistribution between in-plane and out-of-plane orbitals while preserving the integrity of electronic states of the trilayer unlike other interface control methods such as ion irradiation ${ }^{26}$ or thermal annealing technique ${ }^{27}$, which may cause a permanent atomic rearrangement and thus induce undesired extrinsic effects. To discuss this temperature dependence of the DMI, that of the spin $\left(\boldsymbol{m}_{\mathrm{s}}\right)$ and orbital $\left(\boldsymbol{m}_{\mathrm{o}}\right)$ magnetic moments of Co and $\mathrm{Pt}$ is studied by X-ray magnetic circular dichroism (XMCD) spectroscopy. We find that $\boldsymbol{m}_{\mathrm{s}}$ values of Co and $\mathrm{Pt}$ show temperature dependences due to change in Heisenberg exchange. Furthermore, the intra-atomic magnetic dipole moment $\left(\boldsymbol{m}_{\mathrm{D}}\right)$, which is due to the asymmetric spin density distribution $\left(f_{\mathrm{SD}}\right)^{28,29}$, displays strong temperature dependence, suggesting a sizable modification of the charge distribution between the in-plane and the out-of-plane $d$-orbitals under temperature variation. We also find that the out-of-plane orbital moment $\left(\boldsymbol{m}_{0}^{\perp}\right)$ shows large temperature dependence while in-plane orbital moment $\left(\boldsymbol{m}_{\mathrm{o}}^{\|}\right)$does not, revealing a close connection between the anisotropy of $\boldsymbol{m}_{0}$ (orbital anisotropy) and the DMI. The ab initio and the tightbinding model calculations suggest that the ISB-dependent electron hopping, which gives rise to the asymmetric charge distribution at the interface of the $\mathrm{FM} / \mathrm{HM}$, is a possible microscopic origin of the correlation between the orbital anisotropy and the DMI.

\section{Results}

Temperature dependence of the DMI in the $\mathrm{Pt} / \mathrm{Co} / \mathrm{MgO}$ trilayer. The temperature dependence of the DMI-induced effective field $\left(\boldsymbol{H}_{\mathrm{DMI}}\right)$ of the $\mathrm{Pt}(2 \mathrm{~nm}) / \mathrm{Co}(0.5 \mathrm{~nm}) / \mathrm{MgO}(2$ $\mathrm{nm})$ trilayer is determined by measuring the nucleation field $\left(\boldsymbol{H}_{\mathrm{n}}\right)$ applied along the in-plane $\left(\boldsymbol{H}_{x}\right)$ and out-of-plane $\left(\boldsymbol{H}_{z}\right)$ direction as schematically shown in Fig. 1a. The measured $\boldsymbol{H}_{\mathrm{n}}$ values are analysed with the extended droplet model ${ }^{24}$. Since $\boldsymbol{H}_{\mathrm{n}}$ is proportional to square of DW energy $\left(\sigma_{\mathrm{DW}}^{2}\right)$, the $\sigma_{\mathrm{DW}}$ is a crucial parameter for the nucleation ${ }^{24,30}$. In case of the droplet with sizable DMI, DW magnetizations are aligned in the radial direction. When we consider the two DW magnetizations with respect to $\boldsymbol{H}_{x}$, DW1 and DW2 (see the inset of Fig. 1b), $\sigma_{\mathrm{DW}}^{2}$ of the DW1 and the DW2, respectively, follows the blue and the red curves in terms of $\boldsymbol{H}_{x}$ due to DMI as illustrated in Fig. $1 b^{31}$
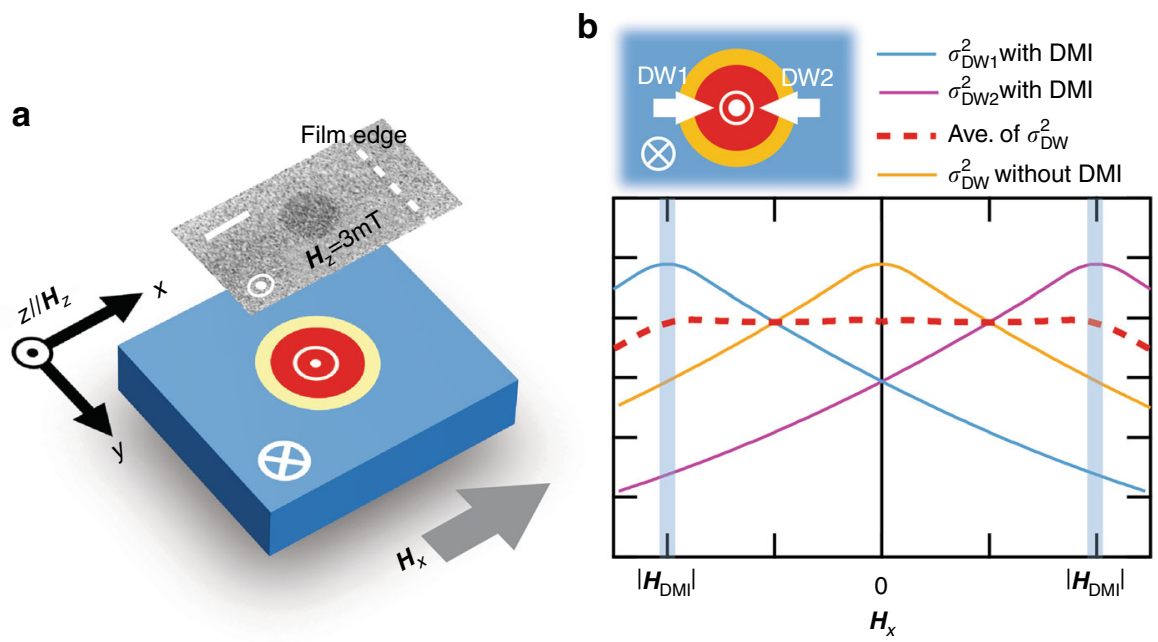

Fig. 1 Droplet nucleation with DMI. a Schematic image of a magnetic droplet in a ferromagnetic medium under $\boldsymbol{H}_{z}$ and $\boldsymbol{H}_{x}$. Inset shows a magneto-optical Kerr effect (MOKE) image to prove the droplet nucleation inside the Pt/Co/MgO microstrip. The white bar is a scale bar of $5 \mu \mathrm{m}$. $\mathbf{b} \mathrm{Schematic} \mathrm{diagrams} \mathrm{of}$ $\sigma_{\mathrm{DW}}^{2}$ in terms of $\boldsymbol{H}_{\mathrm{x}}$. Inset shows the magnetization alignments of the DW1 and DW2 in the DW of the droplet. The thresholds in the curve of averaged $\sigma_{\mathrm{DW}}^{2}$ are highlighted with blue shades 
Assuming that total DW energy mainly depends on the two magnetizations at the DW of a droplet with respect to $\boldsymbol{H}_{x}$, the total $\sigma_{\mathrm{DW}}^{2}$ follows the trend of the purple-dotted line. Note that there is a threshold where the $\sigma_{\mathrm{DW}}^{2}$ starts to become $\boldsymbol{H}_{x}$ dependent. Our previous report showed that the extended droplet model allows us to estimate the DMI-induced field from the threshold field ${ }^{24}$. In spite of such one-dimensional approximation, we have demonstrated that the fitting gives reliable value of the DMI energy density $(D)$.

The measured $\boldsymbol{H}_{\mathrm{n}}$ as a function of $\boldsymbol{H}_{x}$ shows a threshold arising from the DMI as predicted by the extended droplet model ${ }^{24}$ (see also Supplementary Note 1,2 and Method section for details about the analysis and the measurement). Figures $2 \mathrm{a}-\mathrm{d}$ show $\boldsymbol{H}_{\mathrm{n}} / \boldsymbol{H}_{\mathrm{SW}}\left(\boldsymbol{H}_{x}=0\right)$ as a function of $\boldsymbol{H}_{x} / \boldsymbol{H}_{K}$ at various temperatures (a, $T=300 \mathrm{~K} ; \mathrm{b}, 200 \mathrm{~K} ; \mathrm{c}, 150 \mathrm{~K}$ and d, $100 \mathrm{~K}$ ). Here $\boldsymbol{H}_{\mathrm{n}}$ and $\boldsymbol{H}_{x}$ are normalized by the switching field at $\boldsymbol{H}_{x}=0\left[\boldsymbol{H}_{\mathrm{SW}}\left(\boldsymbol{H}_{x}=0\right)\right]$ and by the anisotropy field $\left(\boldsymbol{H}_{K}\right)$, respectively. Those normalized values allow us to clearly confirm the DMI-dependent threshold with ruling out the temperature-dependent characteristics of $\boldsymbol{H}_{K}$ and the $\boldsymbol{H}_{\mathrm{SW}}\left(\boldsymbol{H}_{x}=0\right)$. The temperature-dependent $\boldsymbol{H}_{\mathrm{DMI}}$ can be determined from the best fitting using the extended droplet model; $\boldsymbol{H}_{\mathrm{DMI}}$ at $300,200,150$ and $100 \mathrm{~K}$ are $166 \pm 50,245 \pm 45,324$ \pm 15 and $372 \pm 30 \mathrm{mT}$, respectively (Fig. 2e). The temperaturedependent $\boldsymbol{D}$ is readily calculated from $\boldsymbol{H}_{\mathrm{DMI}}$ and $\Delta$ via $\boldsymbol{H}_{\mathrm{DMI}}=\boldsymbol{D} / \mu_{0} M_{\mathrm{s}}$, where $\mu_{0}$ is the permeability, $M_{\mathrm{s}}$ is the saturation magnetization and $\Delta$ is the DW width ${ }^{6} . \Delta$ and $M_{\mathrm{s}}$ values in terms of the temperature are listed in Table 1 . We find that $\boldsymbol{D}$ has a strong temperature dependence as shown in Fig. 2e; $D$ increases by a factor of 2.2 as the temperature decreases from 300 to $100 \mathrm{~K}$.

Heisenberg exchange is one of key parameters to understand the microscopic origin of $\mathrm{DMI}^{2,32}$. From the temperature dependence of $M_{\mathrm{s}}$, the exchange stiffness constant $(A)$ values were quantitatively obtained as detailed in Supplementary Note 3.
As listed in Table 1, we find a clear correlation of the Heisenberg exchange with DMI; the $A$ value increases by $57 \%$ when the temperature decreases from $300 \mathrm{~K}$ to $100 \mathrm{~K}$ while DMI shows $100 \%$ increase. Based on Moriya's theory, DMI requires three ingredients: (i) SOC, (ii) magnetic exchange, and (iii) ISB. Therefore, we can consider two parameters in addition to Heisenberg exchange: one is the proximity-induced magnetism of the non-magnetic element, which is related to the source ii, and the other is the hybridization between $3 d$ and $5 d$ orbitals, which is related to the sources $i$ and iii. In order to find how DMI correlated with the induced moment in the Pt layer and the orbital structure of $\mathrm{Co}$, XMCD studies were performed using the soft and hard X-ray as discussed in the following sections.

Temperature dependence of the proximity-induced magnetic moment of Pt. In this section, we first investigate the role of the induced magnetic moment in the Pt layer as mentioned in previous section. The temperature dependence of the Pt-induced magnetic moment was measured using the XMCD method, which enables element-specific analyses of spin and orbital magnetism ${ }^{33,34}$. Figure 3a presents the XMCD and integration of XMCD spectra measured at the Pt $L_{2,3}$ edge. The intensities of XMCD are $~ 3 \%$ of the X-ray absorption spectra (XAS) edge heights. At both the $L_{3}$ and $L_{2}$ edges (around $11.57 \mathrm{keV}$ and 13.28 $\mathrm{keV}$, respectively), the integrated XMCD spectra show temperature dependences. In contrast, there is no temperature dependence in XAS spectra (see inset of Fig. 3a). The total magnetic moment $\left(\boldsymbol{m}_{\text {total }}\right)$, which is the sum of an effective spin magnetic moment $\left(\boldsymbol{m}_{\mathrm{s}}^{\text {eff }}=\boldsymbol{m}_{\mathrm{s}}+\boldsymbol{m}_{\mathrm{D}}\right)$ and $\boldsymbol{m}_{\mathrm{o}}$, was estimated by a sum rule calculation ${ }^{35}$ (see the details about the sum rule calculation in Supplementary Note 4). In this study, we use moment values per single hole rather than those per atom because it is difficult to precisely determine the hole number $\left(n_{\mathrm{h}}\right)$ of $\mathrm{Pt}$ in the $\mathrm{Pt} / \mathrm{Co} /$ $\mathrm{MgO}$. The moment values normalized by $n_{\mathrm{h}}$ are directly obtained
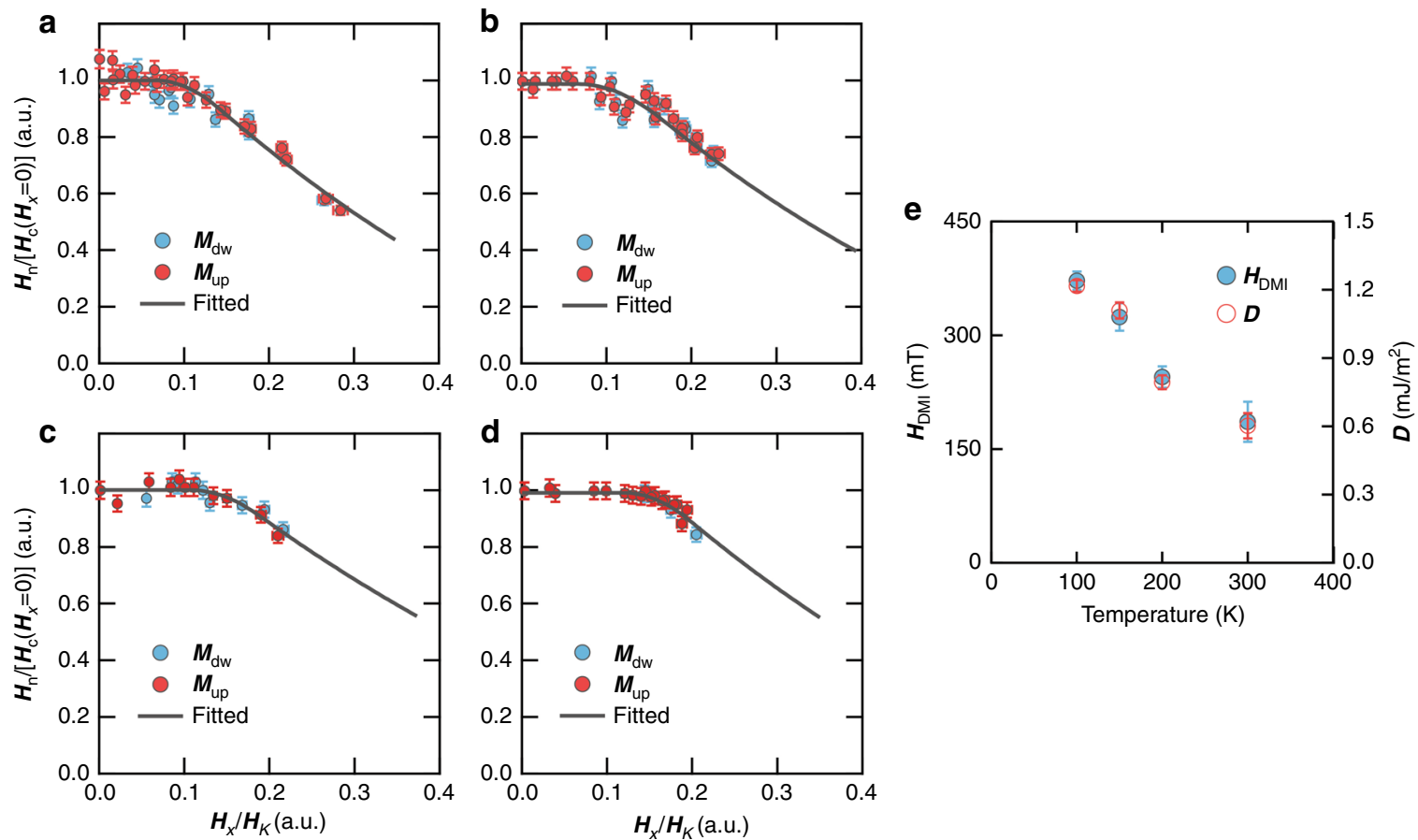

Fig. $2 \boldsymbol{H}_{\mathrm{DMI}}$ measurement from the $H_{\mathrm{n}}$ of the droplet. a-d The $\boldsymbol{H}_{\mathrm{n}} / \boldsymbol{H}_{\mathrm{sw}}\left(\boldsymbol{H}_{x}=0\right)$ vs $\boldsymbol{H}_{x} / \boldsymbol{H}_{K}$ plots measured at 300, 200, 150 and $100 \mathrm{~K}$, respectively. The grey solid lines are the best fitting results using the droplet model. Here the vertical axis is normalized by the nucleation field $\boldsymbol{H}_{\mathrm{sw}}\left(\boldsymbol{H}_{x}=0\right)$ at $\boldsymbol{H}_{x}=0$ and the horizontal axis is normalized by the effective perpendicular anisotropy field $\boldsymbol{H}_{K}$. $\mathbf{e}$ Plots of $\boldsymbol{H}_{\mathrm{DMI}}$ and $\boldsymbol{D}$ in terms of $T$. The error bars are based on the standard deviation of the $\boldsymbol{H}_{\mathrm{DMI}}$ distribution from the best fitting 
Table 1 Parameters to estimate the DW energy

$\begin{array}{lllllll}\text { Temperature } & M_{\mathrm{s}}(\mathrm{MA} / \mathrm{m}) & K_{\mathrm{U}}\left(\mathrm{MJ} / \mathrm{m}^{3}\right) & A(\mathrm{pJ} / \mathrm{m}) & \Delta(\mathrm{nm}) & K_{\mathrm{D}}\left(10 \mathrm{~kJ} / \mathrm{m}^{3}\right) & \sigma_{0}\left(\mathrm{~mJ} / \mathrm{m}^{2}\right) \\ 300 & 1.06 & 0.83 \pm 0.01 & 5.85 \pm 0.1 & 2.7 \pm 0.1 & 9.9 \pm 0.4 & 8.80 \pm 0.4 \\ 200 & 1.22 & 1.26 \pm 0.01 & 7.76 \pm 0.1 & 2.5 \pm 0.1 & 9.82 \pm 2.0 & 12.5 \pm 0.3 \\ 150 & 1.28 & 1.37 \pm 0.01 & 8.81 \pm 0.1 & 2.5 \pm 0.1 & 9.82 \pm 2.0 & 13.9 \pm 0.2 \\ 100 & 1.33 & 1.60 \pm 0.20 & 9.22 \pm 0.1 & 2.4 \pm 0.2 & 9.15 \pm 2.0 & 15.4 \pm 0.2\end{array}$

$\Delta$, the domain wall anisotropy $\left(K_{\mathrm{D}}\right)$, and the Bloch-type DW energy $\left(\sigma_{0}\right)$ values obtained from the best fitting of $\boldsymbol{H}_{\mathrm{n}}\left(\boldsymbol{H}_{x}\right) /\left[\boldsymbol{H}_{\mathrm{sw}}\left(\boldsymbol{H}_{\mathrm{x}}=0\right)\right]$ vs $\boldsymbol{H}_{x} / \boldsymbol{H}_{\mathrm{K}}$ plots using the extended droplet model. $K_{\mathrm{D}}$ is $\mathrm{DW}$ anisotropy energy, representing the magnetostatic energy difference between Bloch DW and Néel DW ${ }^{41}$. The values are comparable with previous reported values in ref. ${ }^{28}$. $M_{\mathrm{s}}$ values were measured using a superconducting quantum interference device magnetometer. Details about estimation of $A$ are given in Supplementary Note 3 .

from the sum rule formula without considering $n_{\mathrm{h}}{ }^{35}$. The changes in the induced magnetic moments with temperature are small $(\sim 15 \%)$ and comparable to the error range of the analysis as shown in Fig. 3b. This suggests that there is a weak correlation between the temperature dependences of the proximity-induced magnetic moments of the Pt layer and the $\mathrm{DMI}$ in the $\mathrm{Pt} / \mathrm{Co} / \mathrm{MgO}$ system ${ }^{11}$.

Correlation between asymmetric orbital structure of the FM layer and the DMI. In this section, we study the correlation of DMI with various physical quantities associated with the magnetism of Co such as $\boldsymbol{m}_{\mathrm{s}}, \boldsymbol{m}_{\mathrm{o}}^{\|}$and $\boldsymbol{m}_{\mathrm{o}}^{\perp}$ and the intra-atomic dipole moment $\boldsymbol{m}_{\mathrm{D}}$. As explained in the previous section, $\boldsymbol{m}_{\mathrm{s} \text { eff }}$ is the sum of $\boldsymbol{m}_{\mathrm{s}}$ and $\boldsymbol{m}_{\mathrm{D}}$. The $\boldsymbol{m}_{\mathrm{s}}$ and $\boldsymbol{m}_{\mathrm{D}}$ values in terms of temperature were obtained from the relation $\boldsymbol{m}_{\mathrm{D}}(\theta)=$ $\boldsymbol{m}_{\mathrm{D}}\left(0^{\circ}\right) \cdot\left(1-3 \cos ^{2} \theta\right), \quad$ thereby $\quad \boldsymbol{m}_{\mathrm{s}, \mathrm{eff}}(\theta)=\boldsymbol{m}_{\mathrm{s}}+\boldsymbol{m}_{\mathrm{D}}\left(0^{\circ}\right)$. $\left(1-3 \cos ^{2} \theta\right)^{28,34}$.

Details about the sum rule calculation are explained in Supplementary Note 4. The XAS and XMCD of the film are measured at 100,200 and $300 \mathrm{~K}$. Two incident angles $\left(\theta=0^{\circ}\right.$ and $70^{\circ}$ with respect to the film normal) were used to separately estimate $\boldsymbol{m}_{\mathrm{o}}^{\|}$and $\boldsymbol{m}_{\mathrm{o}}^{\perp}$ values using the relation $\boldsymbol{m}_{\mathrm{o}}(\theta)=\boldsymbol{m}_{\mathrm{o}}^{\perp} \cos ^{2} \theta+\boldsymbol{m}_{\mathrm{o}}^{\|} \sin ^{2} \theta^{36}$. Figure $4 \mathrm{a}, \mathrm{b}$ are typical XAS and XMCD spectra at the Co $L_{2,3}$ edges obtained at $0^{\circ}$ and $300 \mathrm{~K}$. The XMCD spectra show a clear temperature dependence of their intensity at both $0^{\circ}$ and $70^{\circ}$ as shown in Fig. $4 \mathrm{c}-\mathrm{f}$. In case of the $\boldsymbol{m}_{\mathrm{s}}$, it increases by $20 \%$ (from $0.87 \mu_{\mathrm{B}} / \mu_{\mathrm{h}}$ to $1.11 \mu_{\mathrm{B}} / n_{\mathrm{h}}$ ) as temperature decreases from 300 to $100 \mathrm{~K}$ (see Fig. $4 \mathrm{~g}$ ), which is consistent with the observed temperature dependence of $\boldsymbol{M}_{\mathrm{s}}$.

In addition to $\boldsymbol{m}_{\mathrm{s}}, \boldsymbol{m}_{\mathrm{D}}$ shows a strong temperature dependence. The $\boldsymbol{m}_{\mathrm{D}}$ reflects the anisotropy of $f_{\mathrm{SD}}$ distorted by the SOC or the crystal-field effect. ${ }^{28,29}$. When the electron occupation becomes asymmetric, for instance, more electrons in the in-plane orbitals than the out-of-plane orbitals, the situation gives rise to both non-zero $\boldsymbol{m}_{\mathrm{D}}$ term and asymmetry in the charge distribution. As a result, asymmetry of the charge distribution naturally implies non-vanishing $f_{\mathrm{SD}}$ at transition metals' interfaces. As a result, the value of $\boldsymbol{m}_{\mathrm{D}}$ determined from the sum rule analysis increases from $0.014 \mu_{\mathrm{B}} / n_{\mathrm{h}}$ to $0.094 \mu_{\mathrm{B}} / n_{\mathrm{h}}$ (Fig. $4 \mathrm{~g}$ ). In addition, the orbital anisotropy also increases as temperature decreases; $\boldsymbol{m}_{o}^{\perp}$ increases from $0.058 \mu_{\mathrm{B}} / n_{\mathrm{h}}$ to $0.080 \mu_{\mathrm{B}} / n_{\mathrm{h}}$, whereas $\boldsymbol{m}_{\mathrm{o}}^{\|}$slightly decreases from $0.044 \mu_{\mathrm{B}} / n_{\mathrm{h}}$ to $0.039 \mu_{\mathrm{B}} / n_{\mathrm{h}}$ (Fig. $4 \mathrm{~h}$ ). These results imply a correlation of DMI with $\boldsymbol{m}_{\mathrm{D}}$ and orbital anisotropy. Since the variation of the perpendicular component of the $m_{\mathrm{o}}$ is also related to magnetocrystalline anisotropy $\left(\boldsymbol{K}_{\mathrm{U}}\right)$ based on the Bruno theory $^{37}$, the correlation between DMI and $K_{U}$ is also reasonable (see the Supplementary Note 5$)$. Figure 5 a shows $\boldsymbol{m}_{\mathrm{o}}^{\perp} / \boldsymbol{m}_{\mathrm{o}}^{\|}(=[$ $\left.\left.\boldsymbol{m}_{\mathrm{o}}^{\perp}(T) / \boldsymbol{m}_{\mathrm{o}}^{\|}(T)\right] /\left[\boldsymbol{m}_{\mathrm{o}}^{\perp}(300 \mathrm{~K}) / \boldsymbol{m}_{\mathrm{o}}^{\|}(300 \mathrm{~K})\right]\right)$, and $\boldsymbol{m}_{\mathrm{D}}(T) / \boldsymbol{m}_{\mathrm{D}}(300 \mathrm{~K})$ plots as a function of the normalized $\boldsymbol{D}=\boldsymbol{D}=(T) / \boldsymbol{D}(300 \mathrm{~K})$ where $T$ inside of parentheses is the measurement temperature. The ratio $\boldsymbol{m}_{\mathrm{o}}^{\perp} / \boldsymbol{m}_{\mathrm{o}}^{\|}$increases by $53 \%$ as $\boldsymbol{D}$ increases, and $\boldsymbol{m}_{\mathrm{D}}$ also shows a clear correlation with the DMI; $m_{\mathrm{D}}(100 \mathrm{~K}) / \boldsymbol{m}_{\mathrm{D}}(300 \mathrm{~K}) \approx 6.4$. Because both orbital anisotropy and $\boldsymbol{m}_{\mathrm{D}}$ are closely related to the orbital occupation with ISB $^{28,37,38}$, these results suggest that the temperature dependence of DMI is governed by the change in asymmetric electron occupation in orbitals in addition to spin magnetic moments as we discuss with the following theoretical studies. We also note that peak intensity of the XAS spectra reflects the hole number in $3 d$ orbitals. We found small change in the absorption intensity when varying the temperature. Quantitatively, difference in the XAS intensity integral ( $\left.I_{\mathrm{XAS}}\right)$ between 300 and $100 \mathrm{~K}$ is about $4 \%\left\{\left[I_{\mathrm{XAS}}(300 \mathrm{~K})-I_{\mathrm{XAS}}(100 \mathrm{~K})\right] /\right.$ $\left.I_{\mathrm{XAS}}(300 \mathrm{~K})=0.04\right\}$. Hence, this indicates that there is a small change in the hole number within the temperature range, reflecting temperature dependence of the charge distribution in orbitals. Nonetheless, the value is so small that it does not affect the trend in the respective quantities normalized by $n_{\mathrm{h}}$ from the sum rule calculation.

Theoretical consideration about the microscopic origin of the DMI. In order to support the insights obtained from our experimental study, we carry out two types of theoretical calculations: a tight-binding model calculation and ab initio calculation based on density function theory (DFT), and the main result is shown in Fig. 5b, c. The details of these two complementary theoretical studies can be found in Supplementary Notes 6 and 7.

For the tight-binding model, we extend the trimer model suggested by Kashid et al. ${ }^{10}$, which contains two magnetic atoms coupled to a spin-orbit coupled non-magnetic ion. Compared to Kashid's trimer model, we add one more orbital $\left(d_{x z}\right)$ on the nonmagnetic site, enabling the computation of the orbital anisotropy (see details in Supplementary Note 6). In order to describe the temperature dependence of parameters in our trimer model, we assume the level broadening increases with the temperature. This broadening can be phenomenologically explained by magnetization fluctuations and the electron-phonon interaction given by atomic vibrations. As this model calculation is too simple, we do not aim to give a quantitative explanation of the experimental data but provide a qualitative understanding of the experimental result.

Figure 5b shows that the tight-binding model calculation reproduces qualitatively our experimental observations; both the DMI and $\boldsymbol{m}_{\mathrm{o}}^{\perp}$ decrease with temperature, while the $\boldsymbol{m}_{\mathrm{o}}^{\|}$is almost temperature independent. Note that the $\boldsymbol{m}_{\mathrm{o}}^{\perp}$ and $\boldsymbol{m}_{\mathrm{O}}^{\mathrm{\rho}}$ cases are related to electron hopping with and without ISB, respectively (Supplementary Note 6). In addition, the ratio of the spin density distribution $\left(f_{\mathrm{SD}}^{\perp} / f_{\mathrm{SD}}^{\|}\right)$between the in-plane $(x y)$ and out-of-plane $(y z, x z)$ orbital states decreases as temperature increases as shown in the inset in Fig. 5b (In our tight-binding model, the spin distribution is defined as $S D_{n}=f_{n, \uparrow}-f_{n, \downarrow}$, where $f_{n, \uparrow}\left(f_{n, \downarrow}\right)$ is the occupation of spin up (down) state and $n$ represents the orbital quantum state $d_{x y}^{A}, d_{x y}^{B}, d_{x y}^{C}, d_{y z}^{C}, d_{x z}^{C}$.). This result implies that the spin distribution variation of the orbital states with ISB is key to change of the orbital moment and the DMI, which is in line with the following DFT result.

To examine the correlation between the DMI and the orbital anisotropy in realistic structures, we also perform ab initio calculations for $\mathrm{Pt}(111) / \mathrm{X}$ ultra-thin films, where $\mathrm{X}$ is a $3 d$ 

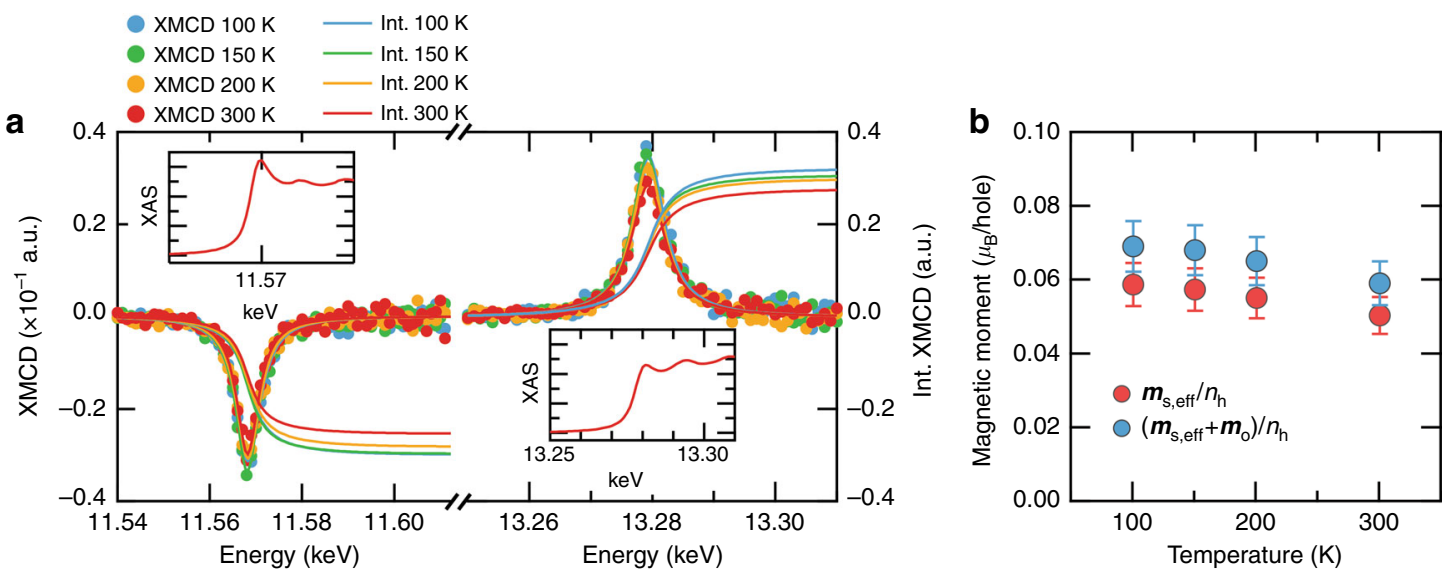

Fig. 3 Temperature dependence of the proximity-induced moment in the Pt layer. a The XMCD and integrated XMCD spectra at the Pt $L_{3}$ and $L_{2}$ edges in terms of temperature. The XMCD spectra were fitted by the Lorentzian function, and the fitted curves were integrated to get the integrated XMCD spectra. The insets are the XAS spectra at the Pt $L_{3}$ and $L_{2}$ edges. $\mathbf{b}$ Temperature dependence of the $\boldsymbol{m}_{\mathrm{s}}^{\text {eff }}$ and $\boldsymbol{m}_{\text {total }}=\boldsymbol{m}_{\mathrm{s}}^{\text {eff }}+\boldsymbol{m}_{\mathbf{o}}$. The error bars are based on the standard deviation of the Lorentzian-fitting residual

transition metal $(\mathrm{X}=\mathrm{V}, \mathrm{Cr}, \mathrm{Mn}, \mathrm{Fe}, \mathrm{Co}, \mathrm{Ni})$. Based on the experimental observation, the microscopic origin of the correlation between the DMI and the orbital anisotropy involves the impact of the temperature on $3 d$-orbital magnetization and their electron filling. In this respect, it is instructive to vary the $3 d$ transition metals on the Pt substrate and examine the general chemical trend. Details about this study are also discussed in Supplementary Note 7 . Figure $5 c$ shows the summary of the DFT calculation. Here changing the overlayer results in modification of the relative alignment between $3 d$ and $5 d$ orbitals and thereby in a modification of the charge distribution: more hybridization results in less asphericity of the charge distribution between the in-plane and the out-of-plane, as reflected by the change in $\boldsymbol{m}_{\mathrm{D}}$. Our calculation results provide a physical insight on how the asymmetric charge distribution induces DMI. As we mentioned in the second section, DMI requires three ingredients: (i) SOC, (ii) magnetic exchange, and (iii) symmetry breaking. While the first two aspects are easily quantifiable, the latter is more difficult to apprehend. It has been reported that the larger the asymmetric spin distribution with symmetry breaking and SOC, the larger intra-dipole spin moment ${ }^{29}$. Therefore, our experimental and theoretical studies propose a method to address the symmetry breaking term. Figure $5 \mathrm{c}$ explains this aspect explicitly. The Mn $3 d$ orbitals and the Pt $5 d$ orbitals provide large magnetic exchange and SOC, respectively. In addition, the dipole moment term becomes maximized with the hybridization between Mn $3 d$ and Pt $5 d$ orbitals. As a result, DMI is maximal. Furthermore, both the DMI and the orbital anisotropy follow the same trend in their signs: $\boldsymbol{D}^{\text {tot }}$ and $\boldsymbol{m}_{\mathrm{o}}^{\perp} / \boldsymbol{m}_{\mathrm{o}}^{\|}$at the $\mathrm{Pt} / \mathrm{V}$ interface have negative sign, while those of other interfaces have positive sign. We also find the correlation between $\boldsymbol{m}_{\mathrm{s}}$ and DMI as confirmed experimentally in this study and in ref. ${ }^{32}$. In contrast, our calculations do not reproduce the experimental correlation reported above between DMI and the induced moment. This may be because of the difference between the actual film stack ( 3 monolayers of $\mathrm{Co})$ and the simulated system ( 1 monolayer of $\mathrm{Co})$. In fact, the total $\mathrm{DMI}$ is dominated by the contribution of the first Co monolayer at the $\mathrm{Co} / \mathrm{Pt}$ interface, while the Heisenberg exchange, which is a source of the induced moment, is affected by the total number of Co overlayers. This is why our calculation, limited to one Co overlayer, well reproduces the correlation of DMI with spin and orbital moments, but not with the induced moment.

Now, the remaining question is how the orbital asphericity can give rise to DMI. At the interface, in-plane orbitals of Co and out- of-plane orbitals of Pt are mixed through inter-atomic hybridization (in our tight-binding model: $d_{x y}$ of Co and $d_{y z}$ of Pt). In the presence of ISB, this hybridization results in a non-vanishing orbital angular momentum: the hopping trajectory of an electron produces an effective orbital angular momentum as illustrated in Fig. 6. Moreover, the large SOC on Pt mixes in-plane and out-ofplane orbitals, which is accompanied by spin canting (in our tight-binding model: $d_{x y}$ and $d_{y z}$ orbitals on Pt site). In other words, the SOC on $\mathrm{Pt}$ converts the interfacial orbital angular momentum of hopping electrons into spin canting, resulting in a non-collinear magnetic texture. In spite of its simplicity, this picture intuitively explains how the orbital asphericity generates the spin canting. A similar idea has been reported in ref. ${ }^{21}$ : the interfacial electric dipole induced by the asymmetric charge distribution results in chiral orbital angular momentum, and thereby spin canting via SOC. Although the latter approach is based on $p$-orbitals, it agrees with the intuitive picture based on $d$ orbitals.

\section{Discussion}

Our experimental study on the temperature dependence of the DMI suggests that the interfacial DMI in FM/HM bilayers originates from Heisenberg exchange and the asymmetric charge distribution caused by the ISB, as evidenced by the orbital anisotropy and magnetic dipole moment $\left(\boldsymbol{m}_{\mathrm{o}}^{\perp} / \boldsymbol{m}_{\mathrm{o}}^{\|}, \boldsymbol{m}_{\mathrm{D}}\right)$. Our DFT simulation and tight-binding calculation provide a clear evidence of the close link between the DMI and orbital physics. Based on the theoretical discussions, the temperature-dependent $\boldsymbol{m}_{\mathrm{D}}$ and $\boldsymbol{m}_{\mathrm{o}}$ indicate that increase in the temperature promotes the phonon-induced electron hopping between the out-of-plane and in-plane orbitals, thereby resulting in a reduced asphericity (or reduced asymmetry) of $f_{\mathrm{SD}}$ over the $d$-orbitals and thus quenching $\boldsymbol{m}_{\mathrm{D}}$.

In our experiment, however, the correlation between $\boldsymbol{m}_{\mathrm{o}}^{\perp} / \boldsymbol{m}_{\mathrm{o}}^{\|}$ and the DMI is only semi-quantitative, i.e. the temperaturedependent change in the DMI does not perfectly scale with the temperature-dependent change in $\boldsymbol{m}_{\mathrm{o}}^{\perp} / \boldsymbol{m}_{\mathrm{o}}^{\|}$. This semiquantitative correlation between the DMI and $\boldsymbol{m}_{\mathrm{o}}$ demands a more detailed discussion. In systems with ISB, $\boldsymbol{m}_{\mathrm{o}}^{\perp}$ can be decomposed into ISB-independent part and ISB-dependent part. Given that ISB is an essential ingredient for the DMI, there should be a direct correlation between the DMI and ISBdependent $\boldsymbol{m}_{\mathrm{o}}$, as evidenced by our tight-binding model 

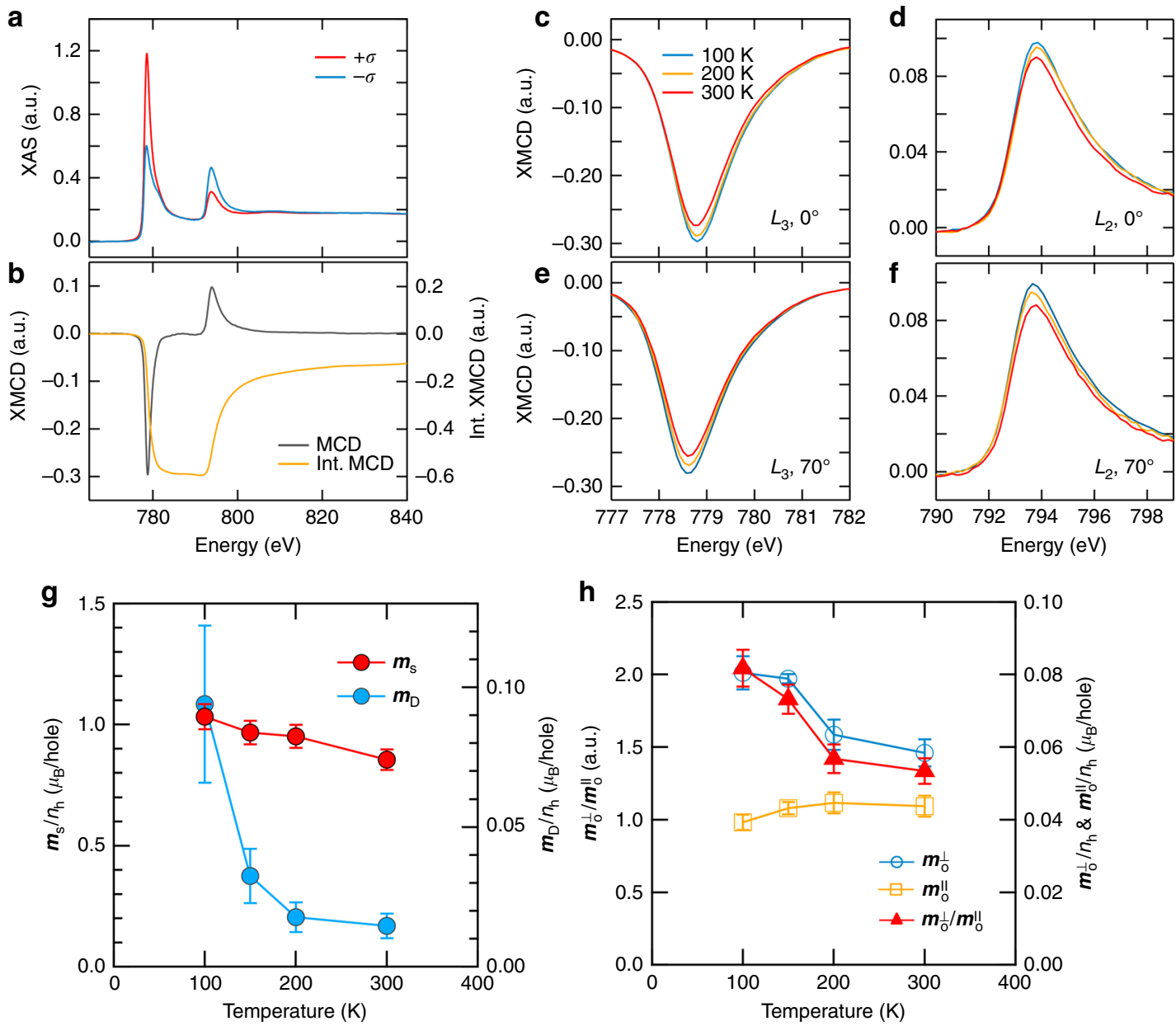

Fig. 4 Temperature dependence of the Co magnetic moments. a XAS spectra for positive $\left(\sigma^{+}\right)$and negative $(\sigma-)$ X-ray helicities. b XMCD and integrated XMCD spectra at $0^{\circ}$ with $300 \mathrm{~K}$. The temperature dependence of XMCD spectra at the $C o L_{3}$ and $L_{2}$ edges measured at $(\mathbf{c}, \mathbf{d}) 0^{\circ}$ and $(\mathbf{e}, \mathbf{f}) 70^{\circ}$. $\mathbf{g}$ Plots of $\boldsymbol{m}_{\mathbf{s}}$ and $\boldsymbol{m}_{\mathrm{D}}$ vs temperature as a function of the X-ray incident angle. $\mathbf{h} \boldsymbol{m}_{\mathrm{o}}^{\perp}, \boldsymbol{m}_{\mathrm{o}}^{\|}$and $\boldsymbol{m}_{\mathrm{o}}^{\perp} / \boldsymbol{m}_{\mathrm{O}}^{\|}$as functions of temperature. The error bars are based on the standard deviation of the integral distribution after subtracting the backgrounds of XAS spectra

calculation. However, $\boldsymbol{m}_{\mathrm{O}}^{\perp}$ also has an ISB-independent part, which precludes a direct and quantitative correlation between the DMI and $\boldsymbol{m}_{\mathrm{o}}^{\perp}$. This statement can be rephrased technically as the DMI involves only off-diagonal elements of the SOC operator ${ }^{10}$, while $\boldsymbol{m}_{\mathrm{o}}$ involves all of them. We note, however, that even with this uncertainty, the experimentally observed correlation between the DMI and $\boldsymbol{m}_{\mathrm{o}}$ anisotropy for the $\mathrm{Pt} / \mathrm{Co} / \mathrm{MgO}$ structure is rather clear, implying that the ISB-dependent $\boldsymbol{m}_{\mathrm{o}}$ would dominate over the ISB-independent one in this structure. Therefore, our findings based on both experimental and theoretical studies provide a link between orbital physics and spin-orbit-related phenomena such as the DMI, which are essential for spin-orbitronic devices.

\section{Method}

Film preparation and device fabrication. $\mathrm{Si} / \mathrm{Ta}(1.5) / \mathrm{Pt}(2) / \mathrm{Co}(0.5) / \mathrm{MgO}(2) /$ $\mathrm{HfO}(5)$ (in $\mathrm{nm}$ ) film with perpendicular magnetic anisotropy was deposited on an undoped Si substrate by direct current magnetron sputtering and the atomic layer deposition technique. A 5- $\mu \mathrm{m}$-wide Hall cross-structure were fabricated using the photo lithography and the Ar ion milling. For the XMCD measurement, the same stack film was prepared.

Nucleation field measurement. Angular-dependent coercivity of the $\mathrm{Co} / \mathrm{Pt}$ Hall device was measured to estimate the $\boldsymbol{H}_{\mathrm{n}}$ of the magnetic droplet at 300,200, 150 and $100 \mathrm{~K}$. The angle between magnetic field and the sample normal was varied from $0^{\circ}$ to $89^{\circ}$ rotating the electromagnet. At each angle, magnetic field was swept with in $\pm 0.5 \mathrm{~T}$ to observe the coercivity. Details about this measurement are also discussed in ref. ${ }^{24}$

XMCD measurement. Soft X-ray: Soft X-ray absorption spectra were measured using the total electron yield method with $96 \%$ circularly polarized incident X-rays at the BL25SU at SPring-8. XMCDs at the Co $L_{3}$ and $L_{2}$ edges (in a range between 770 and $840 \mathrm{keV}$ ) were recorded in the helicity-switching mode with an applied magnetic field of 1.9 T. Homogeneity of the magnetic field was better than $99 \%$ for $\phi 10 \mathrm{~mm}$ at the sample position. The incident light direction was inclined by $10^{\circ}$ with respect to the magnetic field direction. Temperature was varied from 300 to $100 \mathrm{~K}$ using a continuous liquid He flow-type cryostat.

Hard X-ray: XMCD experiments using hard X-rays were carried out at BL39XU of SPring-8. A circularly polarized X-ray beam with a high degree of circular polarization $(>95 \%)$ was produced with a transmission-type diamond X-ray phase retarder of 1.4-mm thickness. XAS of the film were observed at a $0.6 \mathrm{~T}$ magnetic field applied parallel to the X-ray propagation direction of which an incident angle was $0^{\circ}$ with respect to the surface normal. The $\mathrm{X}$-ray fluorescence yield mode was used to record the spectra. The X-ray energy was scanned around the Pt $L_{3}$ and $L_{2}$ edges in a range between 11.5 and $3.5 \mathrm{keV}$, reversing the $\mathrm{X}$-ray photon helicity at $0.5 \mathrm{~Hz}$. In this manner, two helicity dependent spectra $\mathrm{I}^{+}$and $\mathrm{I}^{-}$were recorded simultaneously. Here $I^{+}$and $I^{-}$denote the intensities when the incident photon momentum and the magnetization vectors are parallel and antiparallel, respectively. The XMCD spectrum, $\Delta I=I^{+}-I^{-}$, is given by the difference of the two spectra. Detailed experimental set-ups for the soft and hard X-ray MCD measurements are described elsewhere ${ }^{39,40}$

DFT calculation. To understand the behaviour of DMI in $3 d / \mathrm{Pt}(111)$ ultrathin films and its correlation with the orbital moment anisotropy (OMA) and magnetic dipole moment $\left(T_{z}\right)$, we have performed DFT calculations in the local density 

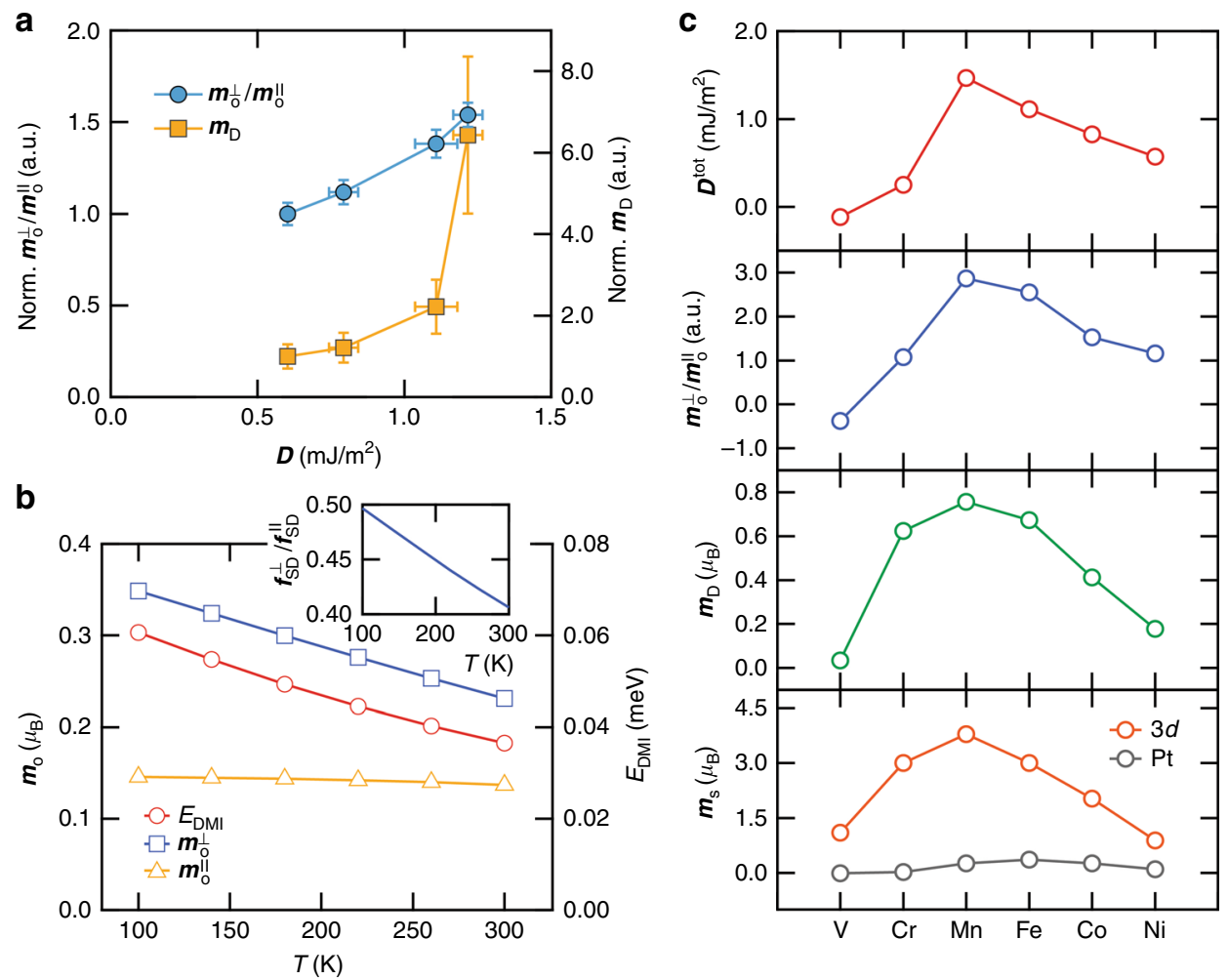

Fig. 5 Correlation of DMI with $\boldsymbol{m}_{\mathbf{o}}^{\perp} / \boldsymbol{m}_{\mathbf{o}}^{\|}$and $\boldsymbol{m}_{\mathbf{D}}$, and theoretical calculations based on the tight binding model and DFT. a Normalized $\boldsymbol{m}_{0}^{\perp} / \boldsymbol{m}_{\mathbf{o}}^{\|}$and $m_{D}$ vs $\boldsymbol{D}$. Values of $\boldsymbol{m}_{\mathrm{o}}^{\perp} / \boldsymbol{m}_{\mathbf{0}}^{\|}$and $\boldsymbol{m}_{\mathrm{D}}$ for all temperatures are normalized by the values measured at $300 \mathrm{~K}$. $\mathbf{b}$ Calculated $\boldsymbol{m}_{\mathrm{o}}^{\perp}, \boldsymbol{m}_{\mathbf{0}}^{\|}$and $E_{\mathrm{DMI}}$ values based on the tightbinding model as a function of temperature. Inset shows $f_{\mathrm{SD}}^{\perp} / f_{\mathrm{SD}}^{\|}$as a function of temperature. c Physical parameters such as total $\boldsymbol{D}\left(\boldsymbol{D}^{\text {tot }}\right), \boldsymbol{m}_{\mathrm{o}}^{\perp} / \boldsymbol{m}_{\mathbf{0}}^{\|} \boldsymbol{m}_{\mathrm{D}}$ and $\boldsymbol{m}_{\mathrm{s}}$ obtained by DFT calculation. Strength and sign of $\boldsymbol{D}^{\text {tot }}$ are calculated around their magnetic ground state using the combination of the relativistic effect spin-orbit coupling with the spin spirals. A positive sign of $\boldsymbol{D}^{\text {tot }}$ indicates a left-rotational sense or left chirality

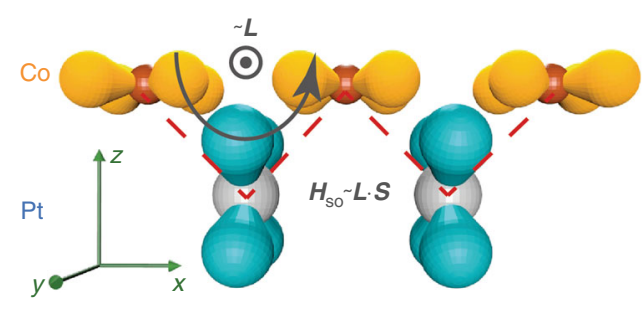

Fig. 6 Schematic illustration of the spin canting. Explicit illustration of the onset of the orbital angular momentum and how SOC induces spin canting from it. The red and grey spheres represent the top ferromagnetic and bottom normal metal atoms in each layer, while the yellow and blue spheres represent the $d_{x y}$ (top) and $d_{y z}$ (bottom) orbitals, respectively. Dotted red lines and black curved arrow indicate the electron hopping path and effective electron orbiting motion due to the hopping, respectively

approximation ${ }^{41}$ to the exchange correlation functional, using the full potential linearized augmented plane wave method in film geometry ${ }^{42}$ as implemented in the Jülich DFT code FLEUR ${ }^{43}$. Both collinear and non-collinear magnetic states have been studied employing an asymmetric film consisting of six substrate layers of $\mathrm{Pt}$ covered by a pseudomorphic $3 d$ monolayer on one side of the film at the distance optimized for the lowest collinear magnetic states. For the non-collinear calculations, we used $p(1 \times 1)$ unit cell applying the generalized Bloch theorem (http://www. flapw.de). The OMA is obtained at relaxed geometry as total energy difference for two different magnetization directions employing the force theorem (the principle axes point along hard and easy axis). We considered 512 and $1024 k$-points in the two-dimensional Brillouin zone (2D-BZ) for the scalar relativistic computation and the calculation with SOC treated within first-order perturbation theory, respectively. Note that high computational accuracy is required since energy differences between different magnetic configurations are tiny $(\sim \mathrm{meV})$ in the present case.
In order to investigate the DMI, first we self-consistently calculate the total energy of homogeneous magnetic spin spirals employing the generalized Bloch theorem within the scalar-relativistic approach ${ }^{44}$. We have considered the energy dispersion $E(q)$ of planar spin spirals, which are the general solution of the Heisenberg Hamiltonian, i.e. states in which the magnetic moment of an atom site $R_{i}$ is given by $M_{i}=M\left[\cos \left(q \cdot R_{i}\right), \sin \left(q \cdot R_{i}\right), 0\right]$ where $q$ is the wave vector propagation of the spin spiral. By imposing the Néel spin spirals along the high symmetry lines of $2 \mathrm{D}-\mathrm{BZ}$, we can scan all possible magnetic configurations that can be described by a single $q$-vector. So, varying the $q$-vector with small steps along the paths connecting the high symmetry points, we find the well-defined magnetic phases of the hexagonal lattice: FM state at $\bar{\Gamma}$-point $(q=0)$, anti-FM state at the $\bar{M}$-point, and periodic $120^{\circ}$ Néel state the $\overline{\mathrm{K}}$-point. When the energy $E(q)$ along the high symmetry lines of $2 \mathrm{D}-\mathrm{BZ}$ is lower than any of the collinear magnetic phases studied previously, the system most likely adopts an incommensurate spin-spiral magnetic ground state structure.

In a second step, we evaluate the DMI contribution from the energy dispersion of spin spirals by applying the SOC treated within first-order perturbation theory combined with the spin spirals ${ }^{5,42}$. Phenomenologically, the antisymmetric exchange interaction DMI has the typical form $E_{\mathrm{DM}}=\sum \boldsymbol{D}_{i, j} \cdot\left(\boldsymbol{S}_{i} \times \boldsymbol{S}_{j}\right)$, where $\boldsymbol{D}_{i, j}$ is the DM vector that determine the strength and sign if DMI and $S_{i}$ and $S_{j}$ are magnetic spin moments located on neighbouring atomic sites $i$ and $j$, respectively. Considering the Néel-type out-of-plane configuration, the $\boldsymbol{D}_{i, j}$ vector should be oriented in plane and normal to the $q$-vector. Note that DMI term must vanish for both configurations Néel-type in-plane and Bloch-type spin spirals due to symmetry arguments ${ }^{44}$. According to our definition, the vector chirality reads $\boldsymbol{C}=\boldsymbol{C} \boldsymbol{c}=S_{i} \times S_{i+1}$, where the direction of the vector spin chirality $c$ is considered as spin rotation axis. Thus the left-handed (right-handed) spin spiral correspond to $C=+1(C=-1)^{45}$.

Data availability. The data that support the findings of this study are available from the corresponding author upon reasonable request.

Received: 05 July 2017 Accepted: 27 March 2018

Published online: 25 April 2018 


\section{References}

1. Dzyaloshinskii, I. E. Thermodynamic theory of weak ferromagnetism in antiferromagnetic substances. Sov. Phys. JETP 5, 1259-1272 (1957).

2. Moriya, T. Anisotropic superexchange interaction and weak ferromagnetism. Phys. Rev. 120, 91-98 (1960).

3. Mühlbauer, S. et al. Skyrmion lattice in a chiral magnet. Science 323, 915-919 (2009).

4. Yu, X. Z. et al. Real-space observation of a two-dimensional skyrmion crystal. Nature 465, 901-904 (2010).

5. Heide, M., Bihlmayer, G. \& Blügel, S. Dzyaloshinskii-Moriya interaction accounting for the orientation of magnetic domains in ultrathin films: $\mathrm{Fe} / \mathrm{W}$ (110). Phys. Rev. B 78, 140403 (2008). (R).

6. Thiaville, A., Rohart, S., Jué, É., Cros, V. \& Fert, A. Dynamics of Dzyaloshinskii domain walls in ultrathin magnetic films. Europhys. Lett. 100, 57002 (2012)

7. Emori, S., Bauer, U., Ahn, S.-M., Martinez, E. \& Beach, G. S. D. Currentdriven dynamics of chiral ferromagnetic domain walls. Nat. Mater. 12, 611-616 (2013).

8. Fert, A., Cros, V. \& Sampaio, J. Skyrmions on the track. Nat. Nanotech. 8, 152-156 (2013).

9. Bode, M. et al. Chiral magnetic order at surfaces driven by inversion asymmetry. Nature 447, 190-193 (2007).

10. Kashid, V. et al. Dzyaloshinskii-Moriya interaction and chiral magnetism in $3 d-5 d$ zigzag chains: tight-binding model and $a b$ initio calculations. Phys. Rev. B 90, 054412 (2014)

11. Ryu, K.-S. et al. Chiral spin torque arising from proximity-induced magnetization. Nat. Commun. 5, 3910 (2014).

12. Yang, H., Thiaville, A., Rohart, S., Fert, A. \& Chshiev, M. Anatomy of Dzyaloshinskii-Moriya interaction at Co/Pt interfaces. Phys. Rev. Lett. 115, 267210 (2015)

13. Belabbes, A., Bihlmayer, G., Bechstedt, F., Blügel, S. \& Manchon, A. Hund's rule-driven Dzyaloshinskii-Moriya interaction at $3 d-5 d$ interfaces. Phys. Rev. Lett. 117, 247202 (2016).

14. Fert, A. \& Levy, P. M. Role of anisotropic exchange interactions in determining the properties of spin-glasses. Phys. Rev. Lett. 44, 1538 (1980)

15. Fert, A. \& Levy, P. M. Anisotropy induced by nonmagnetic impurities in CuMn spin-glass alloys. Phys. Rev. B 23, 4667 (1981).

16. Fert, A. Magnetic and transport properties of metallic multilayers. Mater. Sci. Forum 59, 439-480 (1990).

17. Kundu, A. \& Zhang, S. Dzyaloshinskii-Moriya interaction mediated by spinpolarized band with Rashba spin-orbit coupling. Phys. Rev. B 92, 094434 (2015).

18. Kim, K.-W. et al. Chirality from interfacial spin-orbit coupling effects in magnetic bilayers. Phys. Rev. Lett. 111, 216601 (2013).

19. Kikuchi, T., Koretsune, T., Arita, R. \& Tatara, G. Dzyaloshinskii-Moriya interaction as a consequence of a Doppler shift due to spin-orbit-induced intrinsic spin current. Phys. Rev. Lett. 116, 247201 (2016).

20. Yamamoto, K. et al. Interfacial Dzyaloshinskii-Moriya interaction and orbital magnetic moments of metallic multilayer films. AIP Adv. 7, 056302 (2017)

21. Park, S. R. et al. Orbital-angular-momentum based origin of Rashba-type surface band splitting. Phys. Rev. Lett. 107, 156803 (2011).

22. Park, J.-H., Kim, C. H., Lee, H.-W. \& Han, J. H. Orbital chirality and Rashba interaction in magnetic bands. Phys. Rev. B 87, 041301(R) (2013).

23. Boulle, $\mathrm{O}$. et al. Room temperature chiral magnetic skyrmions in ultrathin magnetic nanostructures. Nat. Nanotech. 11, 449-454 (2016).

24. Kim, S. et al. Magnetic droplet nucleation with a homochiral Néel domain wall. Phys. Rev. B 95(22), 220402(R) (2017).

25. Giustino, F. Electron-phonon interactions from first principles. Rev. Mod. Phys. 89, 015003 (2017).

26. Sakamaki, M. et al. Perpendicular magnetic anisotropy in a $\mathrm{Pt} / \mathrm{Co} / \mathrm{Pt}$ ultrathin film arising from a lattice distortion induced by ion irradiation. Phys. Rev. B 86, 024418 (2012).

27. Kim, M. et al. Contribution of individual interfaces in the $\mathrm{MgO} / \mathrm{Co} / \mathrm{Pd}$ trilayer to perpendicular magnetic anisotropy upon annealing. Appl. Phys. Lett. 106, 102404 (2015)

28. $\mathrm{Wu}, \mathrm{R}$. \& Freeman, A. J. Limitation of the magnetic-circular-dichroism spin sum rule for transition metals and importance of the magnetic dipole term. Phys. Rev. L 73, 1994 (1994).

29. Šipr, O., Minár, J. \& Ebert, H. Influence of spin-orbit coupling on the magnetic dipole term $\mathrm{T}_{\alpha}$. Phys. Rev. B 94, 144406 (2016).

30. Pizzini, S. et al. Chirality-induced asymmetric magnetic nucleation in $\mathrm{Pt} / \mathrm{Co} /$ AlOx ultrathin microstructures. Phys. Rev. Lett. 113, 047203 (2014).

31. Je, S.-G. et al. Asymmetric magnetic domain-wall motion by the Dzyaloshinskii-Moriya interaction. Phys. Rev. B 88, 214401 (2013).

32. Nembach, H. T., Shaw, J. M., Weiler, M., Jué, E. \& Silva, T. J. Linear relation between Heisenberg exchange and interfacial Dzyaloshinskii-Moriya interaction in metal films. Nat. Phys. 11, 825-829 (2015).
33. Carra, P., Thole, B. T., Altarelli, M. \& Wang, X. X-ray circular dichroism and local magnetic fields. Phys. Rev. Lett. 70, 694 (1993).

34. Stöhr, J. Exploring the microscopic origin of magnetic anisotropies with X-ray magnetic circular dichroism (XMCD) spectroscopy. J. Magn. Magn. Mater. 200, 470-497 (1999).

35. Chen, C. T. et al. Experimental confirmation of the X-ray magnetic circular dichroism sum rules for iron and cobalt. Phys. Rev. B 75, 152 (1995).

36. Stöhr, J. \& König, H. Determination of spin- and orbital-moment anisotropies in transition metals by angle-dependent $\mathrm{x}$-ray magnetic circular dichroism. Phys. Rev. B 75, 3748 (1995).

37. Bruno, P. Tight-binding approach to the orbital magnetic moment and magnetocrystalline anisotropy of transition-metal monolayers. Phys. Rev. B 39, 1 (1989)

38. Oguchi, T. \& Shishidou, T. Anisotropic property of magnetic dipole in bulk, surface, and overlayer systems. Phys. Rev. B 70, 024412 (2004).

39. Nakamura, K. et al. Development of a soft X-ray magnetic circular circular dichroism spectrometer using a 1.9 T electromagnet at BL25SU of SPring-8. J. Electron. Spectrosc. Relat. Phenom. 144, 1035-1038 (2005).

40. Suzuki, M. et al. Depth profile of spin and orbital magnetic moments in a subnanometer Pt film on Co. Phys. Rev. B 72, 054430 (2005).

41. Jung, S. W. et al. Current-induced domain wall motion in a nanowire with perpendicular magnetic anisotropy. Appl. Phys. Lett. 92, 202508 (2008).

42. Perdew, J. P. \& Zunger, A. Self-interaction correction to density-functional approximations for many-electron systems. Phys. Rev. B 23, 5048 (1981).

43. Wimmer, E., Krakauer, H., Weinert, M. \& Freeman, A. J. Full-potential selfconsistent linearized-augmented-plane-wave method for calculating the electronic structure of molecules and surfaces: $\mathrm{O}_{2}$ Molecule. Phys. Rev. B 24, 864 (1981).

44. Kurz, P., Förster, F., Nordström, L., Bihlmayer, G. \& Blügel, S. Ab initio treatment of noncollinear magnets with the full-potential linearized augmented plane wave method. Phys. Rev. B 69, 024415 (2004).

45. Heide, M., Bihlmayer, G. \& Blügel, S. Describing Dzyaloshinskii-Moriya spirals from first principles. Phys. B Cond. Matter 404, 2678-2683 (2009).

\section{Acknowledgements}

We also thank H.-W. Lee for fruitful discussion about the relation between the orbital magnetism and the DMI. This work was partly supported by JSPS KAKENHI Grant Numbers 15H05702, 26870300, 26870304, 26103002 and 25220604; JSPS Postdoctoral Fellowship program (Grant Number 2604316, P16314); Collaborative Research Program of the Institute for Chemical Research, Kyoto University; R \& D project for ICT Key Technology of MEXT from the Japan Society for the Promotion of Science (JSPS) and the Cooperative Research Project Program of the Research Institute of Electrical Communication, Tohoku University. This work has also been performed with the approval of the SPring-8 Program Advisory Committee (Proposal Nos. 2015A0117, 2015A0125). A.M. and A.B. acknowledge support from King Abdullah University of Science and Technology (KAUST) and fruitful discussiojns with S. Blügel and G. Bihlmayer. G.G., P.-H.J. and K.-J.L. also acknowledge support from the National Research Foundation of Korea (NRF-2015M3D1A1070465, 2017R1A2B2006119). K.-J.K. was supported by the National Research Foundation of Korea (NRF) grant funded by the Korea Government (MSIP) (Nos. 2017R1C1B2009686, NRF-2016R1A5A1008184) and by the DGIST R\&D Program of the Ministry of Science, ICT and Future Planning (17-BT-02). S.K. was supported by Priority Research Centers Program through the National Research Foundation of Korea (NRF) funded by the Ministry of Education (2009-0093818)

\section{Author contributions}

S.K., K.U., K.-J.K., T.M. and T.O. conceived and designed the study. S.K., K.U., T.K. and D.C. fabricated the device and performed the nucleation field measurement. S.K., D.-H. K., P.-H.J., T.M., K.-J.K. and T.O. contributed to determine the DMI-induced effective field using the droplet model. S.K., M.S., Y.K., T.N. and K.-J.K. designed and performed XMCD experiment and analysed the data. K.U. and K.Y characterizes the magnetic properties of films. A.B., A.M. and K.N. supported theoretical analyses with ab initio calculation. G.G. and K.-J.L. supported the tight-binding model for the temperature dependence of the DMI and orbital magnetism. All authors discussed the results and wrote the manuscript.

\section{Additional information}

Supplementary Information accompanies this paper at https://doi.org/10.1038/s41467018-04017-x.

Competing interests: The authors declare no competing interests.

Reprints and permission information is available online at http://npg.nature.com/ reprintsandpermissions/ 
Publisher's note: Springer Nature remains neutral with regard to jurisdictional claims in published maps and institutional affiliations.

(c) (i) Open Access This article is licensed under a Creative Commons Attribution 4.0 International License, which permits use, sharing, adaptation, distribution and reproduction in any medium or format, as long as you give appropriate credit to the original author(s) and the source, provide a link to the Creative Commons license, and indicate if changes were made. The images or other third party material in this article are included in the article's Creative Commons license, unless indicated otherwise in a credit line to the material. If material is not included in the article's Creative Commons license and your intended use is not permitted by statutory regulation or exceeds the permitted use, you will need to obtain permission directly from the copyright holder. To view a copy of this license, visit http://creativecommons.org/ licenses/by/4.0/.

(C) The Author(s) 2018 